\title{
Effect of the Grain Size of Powder Targets on the Spectra of Parametric X-Ray Radiation of Relativistic Electrons
}

\author{
V. I. Alekseev ${ }^{a, *}$, A. N. Eliseev ${ }^{a}$, I. A. Kishin ${ }^{a, b}$, A. S. Klyuev ${ }^{a, b}$, \\ A. S. Kubankin ${ }^{a, b}$, and R. M. Nazhmudinov ${ }^{a, b}$ \\ ${ }^{a}$ Lebedev Physical Institute, Russian Academy of Sciences, Moscow, 119991 Russia \\ ${ }^{b}$ Belgorod State National Research University, Belgorod, 308015 Russia \\ *e-mail: rinaskela@gmail.com
}

Received September 5, 2020; revised September 15, 2020; accepted September 16, 2020

\begin{abstract}
The results of measurements of the dependence of the full width at half-maximum and the relative intensity of spectral peaks of parametric X-ray radiation (PXR) for various crystallographic planes on the grain size of a micrometer diamond powder are presented.
\end{abstract}

Keywords: parametric X-ray radiation, powder targets, relativistic electrons

DOI: $10.3103 / \mathrm{S} 1068335621020056$

\section{INTRODUCTION}

Parametric X-ray radiation (PXR) arises due to the interaction of incident charged particles with atomic electron shells of a periodic medium. There is a large number of theoretical studies and reviews on the study of PXR from crystals [1-3], mosaic crystals [4], and polycrystalline media [5, 6]. These theories are well approved and were confirmed in studies of crystals [7, 8], mosaic crystals [9], and polycrystals [10-12].

This study continues PXR studies from powder targets, i.e., targets without preferred grain orientation (without texture). The random grain orientation in powders allows PXR recording from various crystallographic planes in contrast to single crystals and textured polycrystals in which, as a rule, radiation from one crystallographic plane dominates. PXR generation in powder targets was first studied in [13] where PXR spectra were quantitatively measured in a tungsten powder target with a grain size of $0.8 \div 1.7 \mu \mathrm{m}$; good agreement of experimental data with the PXR kinematic theory for polycrystalline media was shown. However, since only one powder type was used, more than as few late as, the dependence of PXR properties on the target grain size was not studied.

As is known, PXR is formed at the attenuation length [14-16] lying in the range from several tens to several hundred micrometers for diamond powder. It is expected that peak broadening should be observed in micrometer powder targets where the grain size is less than the attenuation length. In [17], it was also shown that the peak broadening effect exists as crystallite sizes decreases.

In this study, the first attempt to reveal the dependence of properties of PXR from powder targets on the grain size was undertaken. The following properties were studied: the dependence of the full width at half-maximum of the PXR reflection and the ratio of PXR peak yields on the grain size.

\section{EXPERIMANTAL}

Experiments were performed using the "Rentgen-1" setup [18] at the Laboratory of Electromagnetic Interactions of the High-Energy Physics Department of the Lebedev Physical Institute. As a source of relativistic electrons, the microtron being an injector of the "PAKHRA" synchrotron was used. The accelerated electron energy was $7 \mathrm{MeV}$, the pulse repetition rate was $50 \mathrm{~Hz}$, the pulse duration was $4 \mu \mathrm{s}$. To increase the number of grains involved in emission, the magneto-optical system was adjusted so that the electron beam diameter would be sufficiently large, i.e., $8 \mathrm{~mm}$, with an initial divergence less than $3 \mathrm{mrad}$ in the target position.

Figure 1 shows the experimental configuration. Radiation was detected at observation angles of $150^{\circ}$ and $180^{\circ}$ by Amptek XR-100 FAST SDD semiconductor drift detectors. The detector efficiency in the 


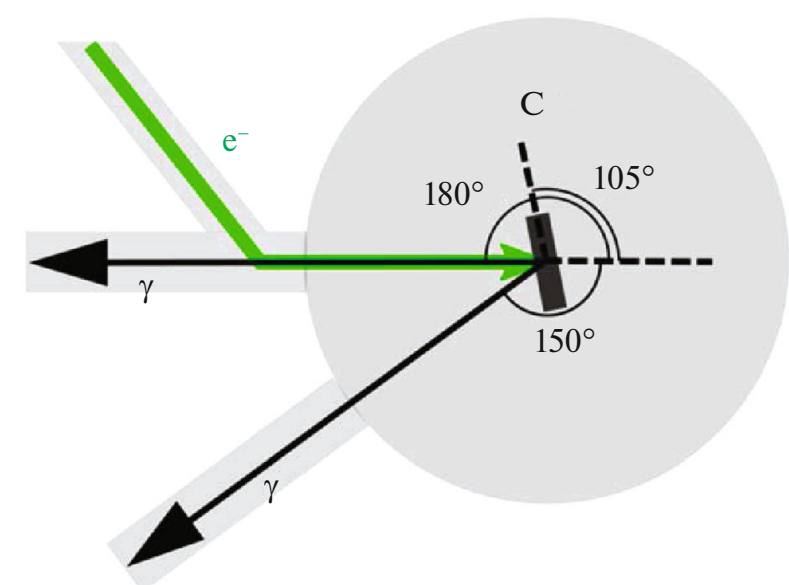

Fig. 1. Experimental configuration: $\mathbf{C}$ is the diamond powder target, $\boldsymbol{\gamma}$ are PXR propagation directions, and e is the electron motion direction.

range from 3 to $10 \mathrm{keV}$ is close to $100 \%$. The solid angles in which radiation was detected for observation angles of $150^{\circ}$ and $180^{\circ}$ with respect to the electrons direction motion were $2 \times 10^{-4}$ sr. compare with and $7 \times 10^{-4} \mathrm{sr}$, respectively.

To perform measurements for diamond powders with grain size of $0.3 \pm 0.1,6 \pm 1$, and $42 \pm 7 \mu \mathrm{m}$, three targets $1 \mathrm{~mm}$ thick were fabricated. The powder was placed between two Mylar films $25 \mu \mathrm{m}$ thick, which were glued on both sides of a polymethylmethacrylate plate with a through rectangular hole $9 \times 23 \mathrm{~mm}^{2}$ in size. At preparation target stages, elementary X-ray analysis of powders and target structural elements was performed. Studies showed that structural elements do not contain impurities with elements harder than oxygen. In diamond powders, silicon, calcium, manganese, chromium, and iron impurities were detected, whose characteristic line energies are not close to PXR energies.

The experimental data obtained are shown in Fig. 2; the red and blue curves correspond to the spectra at observation angles of $150^{\circ}$ and $180^{\circ}$, respectively. The study succeeded to reveal PXR reflections from the (111) plane with energies of 3.01 and $3.12 \mathrm{keV}$ (attenuation lengths are 31.8 and $35.6 \mu \mathrm{m}$ ) and from the (220) plane with energies of 4.916 and $5.089 \mathrm{keV}$ (attenuation lengths are 165.5 and $143.1 \mu \mathrm{m}$ ) for observation angles of $180^{\circ}$ and $150^{\circ}$, respectively; these reflections were revealed for all target types. Reflections from the (311) plane were also detected, but they were close to the characteristic line of manganese (5.97 and $5.9 \mathrm{keV}$, respectively).

There are also spectral characteristic lines of impurity elements incorporated in used powders. An additional confirmation that exactly PXR was observed, rather than characteristic X-ray radiation (CXR), is the dependence of the peak energy on the observation angle; in the case of PXR observation at an angle of $180^{\circ}$, the peak energy was lower than that for the observation angle of $150^{\circ}$.

\section{RESULTS AND DISCUSSION}

For mathematical treatment, a function best describing experimental data was found. It consisted of a sum of a quadratic function for background and from 6 to 10 Gaussian curves one for each distinguished peak. Thus, this became possible to separate contributions of two peaks if they are overlapped.

After mathematical treatment, the ratio of PXR peak areas (red) was determined, which allowed elimination of the error introduced by calibration. Figure 4 shows the found ratios.

As seen in Fig. 4, there is a trend toward changing the ratio of peak areas depending on the grain size; as it increases, the intensity of radiation with higher energies increases, which can be due to the fact that the powder grain size is smaller than the attenuation length for powders 0.3 and $6 \mu \mathrm{m}$ in size for the (111) plane, and is smaller than the attenuation length for all powders for the (220) plane.

Figure 5 shows the full widths at half-maximum of PXR peaks of two planes, and attenuation lengths in diamond for energies 3.01 and $3.12 \mathrm{keV}$ are indicated, which correspond to energies of PXR lines from the (111) plane at observation angles of $180^{\circ}$ and $150^{\circ}$, respectively. The attenuation length in diamond for 


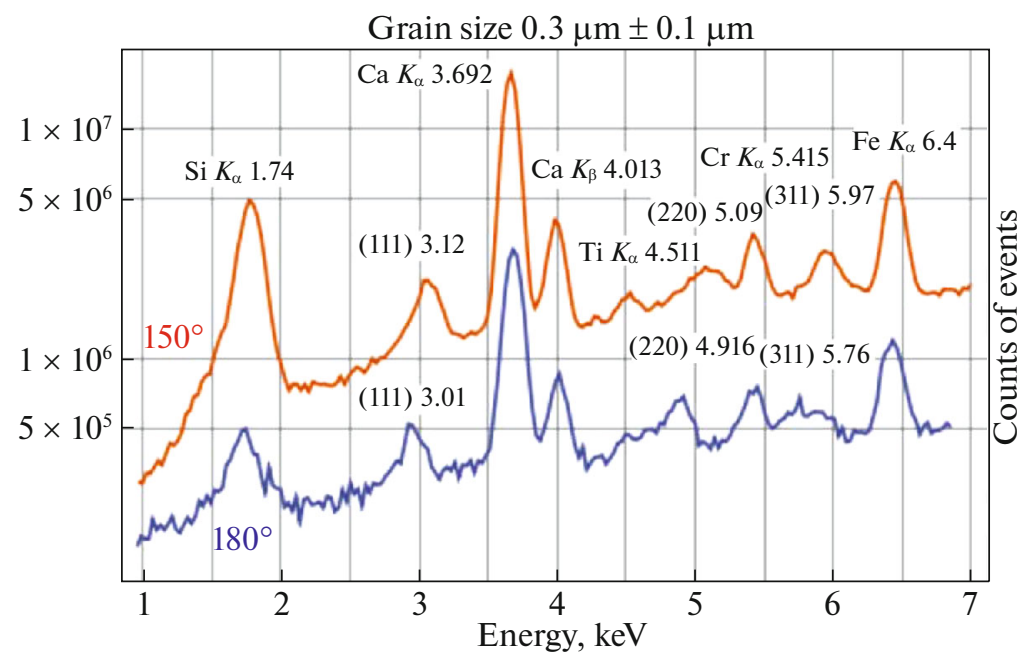

Grain size $6 \mu \mathrm{m} \pm 1 \mu \mathrm{m}$
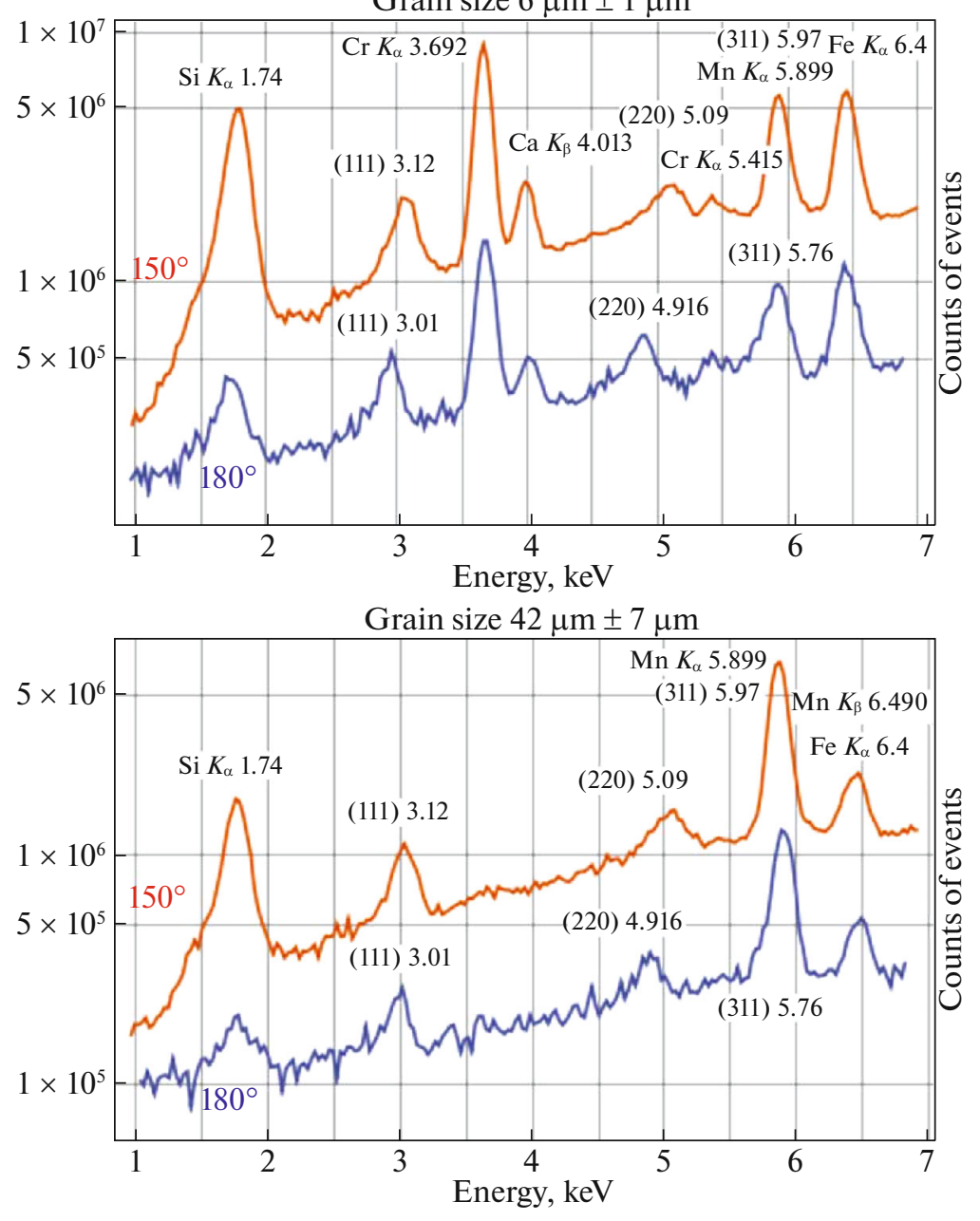

Fig. 2. Spectra of PXR produced in powder targets with grain sizes of $0.3,6,42 \mu \mathrm{m}$, measured at observation angles of $150^{\circ}$ (red curve) and $180^{\circ}$ (blue curve). Peaks with energies near 3 and $5 \mathrm{keV}$ correspond to PXR from (111) and (220) crystallographic planes; other peaks relate to CXR of impurity elements.

energies of 4.92 and $5.09 \mathrm{keV}$ is 143.1 and $165.5 \mu \mathrm{m}$, respectively, which is much large than powder grains of all samples. We can see that the peak width increases for the (111) plane with decreasing grain size, but changes are not observed for the (220) plane. 


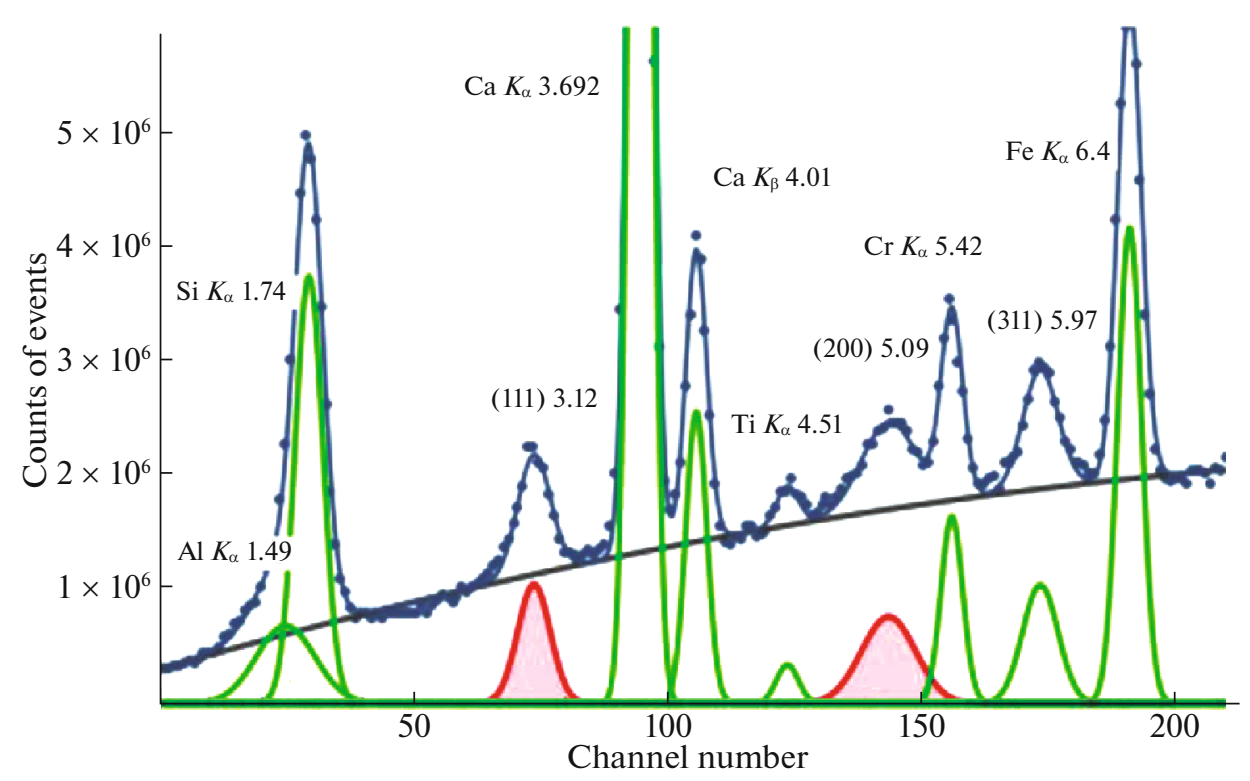

Fig. 3. Spectrum of a diamond powder with a grain size of $0.3 \mu \mathrm{m}$, measured at an observation angle of $150^{\circ}$ (dots), fitted background curve (gray), Gaussian-fitted curves CXR peaks (green), and Gaussian-fitted PXR peaks (red).

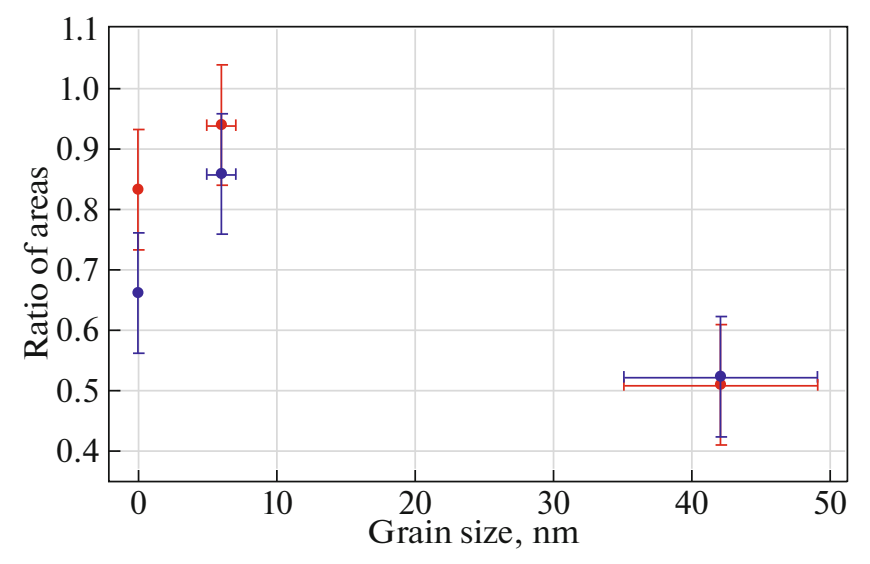

Fig. 4. Ratio of the PXR (111) peak area to the (220) peak area for powders with the various grain sizes: red and blue dots correspond to observation angles of $150^{\circ}$ and $180^{\circ}$, respectively.

\section{CONCLUSIONS}

PXR spectra of relativistic electrons produced in a diamond powder with various grain sizes from 0.3 to $42 \mu \mathrm{m}$ were measured. PXR peaks from crystallographic (111), (220), (331) planes for observation angles of $150^{\circ}$ and $180^{\circ}$ were reliably detected in these spectra.

Mathematical treatment of the experimental results revealed the dependence of the ratio of the PXR yield of the (111) plane that of the (220) plane on the grain size; the intensity of harder radiation increases with the grain size.

The dependences of the peak width on the grain size were obtained. The dependence for the (111) crystallographic plane shows that the PXR spectrum narrows with increasing grain size; for the (220) plane, the dependence on the grain size is not observed. This can be associated with that the attenuation lengths (31.8 and $35.6 \mu \mathrm{m})$ for the (111) plane are comparable to the grain size; for the (220) plane, attenuation lengths are much larger (165.5 and $143.1 \mu \mathrm{m}$ for observation angles of $180^{\circ}$ and $150^{\circ}$, respectively). The next stage will be PXR measurements in powder targets with grain sizes less than $100 \mathrm{~nm}$. 


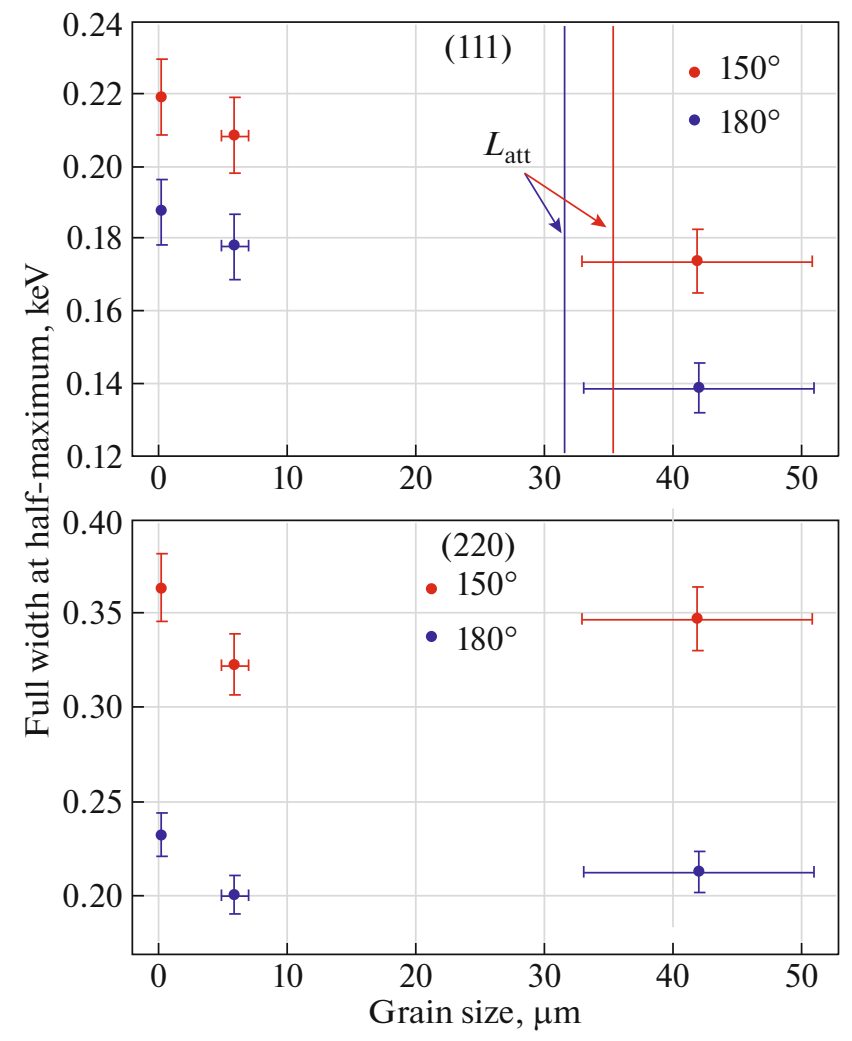

Fig. 5. Full width at half-maximum of PXR peaks of two planes as a function of the grain size and attenuation length in diamond for energies of peaks from the (111) plane (for 3.01 and $3.12 \mathrm{keV}$, blue and red lines, respectively).

\section{FUNDING}

This work was supported by a Program of the Ministry of Science and High Education of the Russian Federation for higher education establishments, project no. FZWG-2020-0032 (2019-1569).

One of the authors was supported by the President of the Russian Federation grant for young scientists and postgraduate students for young scientists and post-graduate students no. SP-765.2019.2.

\section{REFERENCES}

1. M. Ter-Mikaelian, High-Energy Electromagnetic Processes in Condensed Media (Wiley, New York, 1972).

2. A. P. Potylitsyn, M. I. Ryazanov, M. N. Strikhanov, and A. A. Tishchenko, Diffraction Radiation from Relativistic Particles (Springer, Berlin Heidelberg, 2010).

3. V. G. Baryshevsky, I. D. Feranchuk, and A. P. Ulyanenkov, Parametric X-Ray Radiation in Crystals: Theory, Experiments and Applications (Springer, Berlin Heidelberg, 2005).

4. A. S. Kubankin and N. N. Nasonov, "On the Possibility of Using Parametric X-Ray Radiation to Study Anisotropy of a Crystal Mosaic Structure,” J. Surf. Invest.: X-ray Synchrotron Neutron Tech. 2, 317 (2008).

5. N. N. Nasonov, "Collective Effects in the Polarization Bremsstrahlung of Relativistic Electrons in Condensed Media,” Nucl. Instrum. Methods Phys. Res. B 145, 19 (1998).

6. V. Astapenko, N. Nasonov, and P. Zhukova, "Anomalous Peak in the Spectrum of Polarizational Bremsstrahlung from Relativistic Electrons Moving through a Solid Target,” J. Phys. B: At. Mol. Opt. Phys. 40, 1337 (2007). https://doi.org/10.1088/0953-4075/40/7/003

7. B. Sones, R. Danon, and R. Block, "X-Ray Imaging with Parametric X-Rays (PXR) from a Lithium Fluoride (LiF) Crystal,” Nucl. Instrum. Methods A 560, 589 (2006). https://doi.org/10.1016/J.NIMA.2006.01.054

8. Y. N. Adishchev, A. S. Artemov, S. V. Afanasiev, et al., "Detection of Parametric X-Ray Radiation from Moderately Relativistic Protons in Crystals," JETP Lett. 81, 241 (2005).

https://doi.org/10.1134/1.1931007 
9. V. I. Alexeyev, A. N. Eliseyev, E. Irribarra, et al., "Observation of Parametric X-Ray Radiation in an Anomalous Diffraction Region,” Phys. Lett. A 380, 2892 (2016).

10. S. Nawang, I. Endo, M. Iinuma, et al., "Parametric X-ray Study from Textured Molybdenum Polycrystal," J. Phys. Soc. Japan 75, 124705 (2006). https://doi.org/10.1143/JPSJ.75.124705

11. V. I. Alexeyev, A. N. Eliseyev, E. Irribarra, et al., "Evolution of the Characteristics of Parametric X-Ray Radiation from Textured Polycrystals under Different Observation Angles," Phys. Lett. A 382, 503 (2018).

12. V. I. Alekseev, A. N. Eliseev, E. F. Irribarra, I. A. Kishin, A. S. Kubankin, R. M. Nazhmudinov, V. V. Polyanski, V. I. Sergienko, and P. N. Zhukova, "Research of the Polarization Bremsstrahlung of Relativistic Electrons in Polycrystalline Targets,” Nucl. Instrum. Methods Phys. Res. B 342, 47 (2015).

13. V. I. Alekseev, A. N. Eliseyev, E. Irribarra I. A. Kishin, A. S. Klyuev, A. S. Kubankin, R. M. Nazhmudinov, S. V. Trofymenko, and P. N. Zhukova, "Parametric X-Ray Radiation from Powders," Phys. Lett. A 383, 770 (2019).

14. P. N. Zhukova, A. S. Kubankin, N. N. Nasonov, et al., "Modification of the EDXD Method for Diagnosing Structured Media,” Zavod. Lab. Diagn. Mater. 74 (10), 32 (2008).

15. P. N. Zhukova, "Collective Effects in Scattering Processes of the Electromagnetic Field of Relativistic Electrons in Condensed Structured Media," Doctoral Dissertation in Mathematics and Physics (Kursk, 2010).

16. N. N. Nasonov, V. A., Nasonova, and I. G. Popov, "Polarization Bremsstrahlung from Relativistic Electrons Moving in a Small-Grained Medium,” Phys. Atomic Nuclei 64, 966 (2001).

17. A. V. Shchagin, "Possibility of Measuring Nanocrystal Sizes Using Parametric X-Ray Radiation,” Vopr. Atom. Nauki Tekhn. No. 2, 197 (2010).

18. V. I. Alexeyev, V. A. Astapenko, A. N. Eliseyev, E. F. Irribarra, V. A. Karpov, I. A. Kishin, Yu. A. Krotov, A. S. Kubankin, R. M. Nazhmudinov, M. Al-Omari, and S. V. Sakhno, "Investigation into the Mechanisms of X-Ray Generation during the Interaction Between Relativistic Electrons and a Medium by means of the Röntgen-1 Setup,” J. Surf. Invest.: X-Ray Synchrotron Neutron Tech. 11, 694 (2017).

Translated by A. Kazantsev 